\title{
Gradient Vector Flow: A New External Force for Snakes
}

\author{
Chenyang $\mathrm{Xu}$ and Jerry L. Prince \\ Department of Electrical and Computer Engineering \\ The Johns Hopkins University, Baltimore, MD 21218
}

\begin{abstract}
Snakes, or active contours, are used extensively in computer vision and image processing applications, particularly to locate object boundaries. Problems associated with initialization and poor convergence to concave boundaries, however, have limited their utility. This paper develops a new external force for active contours, largely solving both problems. This external force, which we call gradient vector flow $(G V F)$, is computed as a diffusion of the gradient vectors of a gray-level or binary edge map derived from the image. The resultant field has a large capture range and forces active contours into concave regions. Examples on simulated images and one real image are presented.
\end{abstract}

\section{Introduction}

Snakes [10], or active contours, are curves defined within an image domain that can move under the influence of internal forces within the curve itself and external forces derived from the image data. The internal and external forces are defined so that the snake will conform to an object boundary or other desired features within an image. Snakes are widely used in many applications, including edge detection [10], shape modeling [16], segmentation [12], and motion tracking [17].

There are two general types of active contour models in the literature today: parametric active contours [10] and geometric active contours $[1,13]$. In this paper, we focus on parametric active contours, which synthesize parametric curves within an image domain and allow them to move toward desired features, usually edges. Typically, the curves are drawn toward the edges by potential forces, which are defined to be the negative gradient of a potential function. Additional forces, such as pressure forces [4], together with the potential forces comprise the external forces. There are also internal forces designed to hold the curve together

This paper is a condensed version of a paper that will appear in IEEE Trans. on Image Proc. This research was supported by NSF grant MIP9350336. Please address correspondence to prince@jhu.edu. (elasticity forces) and to keep it from bending too much (bending forces).

There are two key difficulties with active contour algorithms. First, the initial contour must, in general, be close to the true boundary or else it will likely converge to the wrong result. The second problem is that active contours have difficulties progressing into concave boundary regions [7]. Although many methods such as multiresolution methods [11], pressure forces [4], distance potential forces [5], control points [7], and using solenoidal external fields [14] have been proposed, they either solve one problem or solve both but creating new difficulties. For example, multiresolution methods have addressed the issue of initialization, but specifying how the snake should move across different resolutions remains problematic. Another example is that of pressure forces, which can push an active contour into boundary concavities, but cannot be too strong or "weak" edges will be overwhelmed [15].

In this paper, we present a new class of external forces for active contour models that addresses the problems listed above. These fields, which we call gradient vector flow (GVF) fields, are dense vector fields derived from images by minimizing an energy functional in a variational framework. The minimization is achieved by solving a pair of decoupled linear partial differential equations which diffuses the gradient vectors of a gray-level or binary edge map computed from the image. We call the active contour that uses the GVF field as its external force a GVF snake. Particular advantages of the GVF snake over a traditional snake are its insensitivity to initialization and ability to move into concave boundary regions.

\section{Background}

\subsection{Parametric Snake Model}

A traditional snake is a curve $\mathrm{x}(s)=[x(s), y(s)], s \in$ $[0,1]$, that moves through the spatial domain of an image to minimize the energy functional

$$
E=\int_{0}^{1} \frac{1}{2}\left(\alpha\left|\mathbf{x}^{\prime}(s)\right|^{2}+\beta\left|\mathbf{x}^{\prime \prime}(s)\right|^{2}\right)+E_{\text {ext }}(\mathbf{x}(s)) d s
$$


where $\alpha$ and $\beta$ are weighting parameters that control the snake's tension and rigidity, respectively. $\mathbf{x}^{\prime}(s)$ and $\mathbf{x}^{\prime \prime}(s)$ denote the first and second derivatives of $\mathbf{x}(s)$ with respect to $s$. The external energy function $E_{\text {ext }}$ is derived from the image so that it takes on its smaller values at the features of interest, such as boundaries.

Given a gray-level image $I(x, y)$ (viewed as a function of continuous position variables $(x, y)$ ), typical external energies designed to lead an active contour toward step edges are [10]:

$$
\begin{aligned}
& E_{\text {ext }}^{1}(x, y)=-|\nabla I(x, y)|^{2} \\
& E_{\text {ext }}^{2}(x, y)=-\left|\nabla\left(G_{\sigma}(x, y) * I(x, y)\right)\right|^{2}
\end{aligned}
$$

where $G_{\sigma}(x, y)$ is a two-dimensional Gaussian function with standard deviation $\sigma$ and $\nabla$ is the gradient operator. If the image is a line drawing (black on white), then appropriate external energies include [4]:

$$
\begin{aligned}
& E_{\mathrm{ext}}^{3}(x, y)=I(x, y) \\
& E_{\mathrm{ext}}^{4}(x, y)=G_{\sigma}(x, y) * I(x, y)
\end{aligned}
$$

It is easy to see from these definitions that larger $\sigma$ 's will cause the boundaries to become blurry and distorted. Such large $\sigma^{\prime}$ 's are often necessary, however, in order to make the effect of the boundary "felt" at some distance from the boundary - i.e., to increase the "capture range" of the active contour.

A snake that minimizes $E$ must satisfy the Euler equation

$$
\alpha \mathbf{x}^{\prime \prime}(s)-\beta \mathbf{x}^{\prime \prime \prime \prime}(s)-\nabla E_{\mathrm{ext}}=\mathbf{0}
$$

This can be viewed as a force balance equation

$$
\mathbf{F}_{\text {int }}+\mathbf{F}_{\text {ext }}^{1}=\mathbf{0}
$$

where $\mathbf{F}_{\text {int }}=\alpha \mathbf{x}^{\prime \prime}(s)-\beta \mathbf{x}^{\prime \prime \prime \prime}(s)$ and $\mathbf{F}_{\mathrm{ext}}^{1}=-\nabla E_{\text {ext }}$. The internal force $\mathbf{F}_{\text {int }}$ discourages stretching and bending while the external potential force $F_{\text {ext }}^{1}$ pulls the snake towards the desired image contour.

To find a solution to (6), the snake is made dynamic by treating $\mathbf{x}$ as function of time $t$ as well as $s-$ i.e., $\mathbf{x}(s, t)$. Then, the partial derivative of $\mathbf{x}$ with respect to $t$ is then set equal to the left hand side of $(6)$ as follows

$$
\mathbf{x}_{t}(s, t)=\alpha \mathbf{x}^{\prime \prime}(s, t)-\beta \mathbf{x}^{\prime \prime \prime \prime}(s, t)-\nabla E_{\text {ext }}
$$

When the solution $\mathrm{x}(s, t)$ stabilizes, the term $\mathbf{x}_{t}(s, t)$ vanishes and we achieve a solution of (6). This dynamic equation can also be viewed as a gradient descent algorithm [3] designed to solve (1). A solution to (7) can be found by discretizing the equation and solving the discrete system iteratively (cf. [10]).

\subsection{Generalized Force Balance Equations}

The external forces generated from the variational formulation (1) must enter the force balance equation (6) as a static irrotational field. ${ }^{1}$ To add additional flexibility to the snake model, it is possible to start from the force balance equation directly, and to replace $F_{\text {ext }}^{1}$ with another force $\mathbf{F}_{\text {ext }}^{2}$, which need not be irrotational, as follows

$$
\mathbf{F}_{\text {int }}+\mathbf{F}_{\text {ext }}^{2}=\mathbf{0}
$$

Balloon models [4] comprise an important example of this approach. In these models $\mathbf{F}_{\text {ext }}^{2}$ is the sum of the traditional potential forces and pressure (or normal) forces, which act in a direction normal to the curve. This increases the capture range of an active contour, but also requires that the balloon be initialized to either shrink or grow. Also, the strength of the pressure forces may be difficult to set, since they must be large enough to overcome weak edges and noise, but small enough so they do not overwhelm legitimate edge forces. In this paper, we consider active contour formulations that do not include pressure forces.

Without pressure forces, two issues become problematic: initialization and convergence to concave regions. Initialization is a problem because the capture range of the traditional potential force is generally small. The capture range can be increased by using a larger $\sigma$, but this blurs and distorts the edges. This difficulty can be addressed using a multiresolution approach, but scheduling changes lead to extremely complex, and ad hoc, algorithms. The distance potential forces of Cohen and Cohen [5] increase the capture range in an effective manner; however, as we have shown in [18] it does not solve the second issue.

Convergence to concave regions is a problem in traditional snakes, because the contour is often left split across boundary concavities. An example of this problem is shown in Fig. 1. Fig. 1a shows a $(64 \times 64$ pixel) line drawing of a $U$-shaped object having a concave region (as viewed from the outside) at the top of the figure, and Fig. 1c shows a sequence of curves depicting the iterative progression of a traditional snake ( $\alpha=0.6, \beta=0.0$, no pressure forces) toward the boundary. The final solution solves the Euler equations of the snake formulation, but remains split across the concave region.

The reason for the poor convergence in Fig. 1c is revealed in Fig. 1b, where the potential force field, $-\nabla E_{\text {ext }}^{4}$ with $\sigma=1.0$ (pixels), for this example is depicted. Although the field correctly points toward the object boundary, in the concave portion the forces point horizontally in opposite directions. Thus, the curve is "pulled" apart toward the U-shape, but not made to progress downward into the concave region. The poor performance in this example lies in

\footnotetext{
${ }^{1}$ An irrotational field is the gradient of a scalar field. Sometimes these fields are called conservative or curl-free fields.
} 


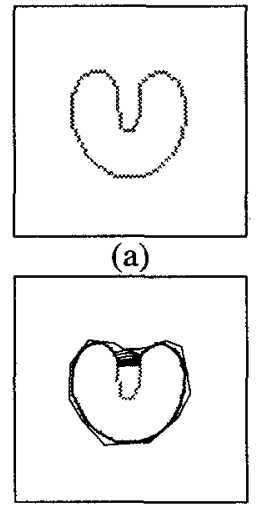

(c)

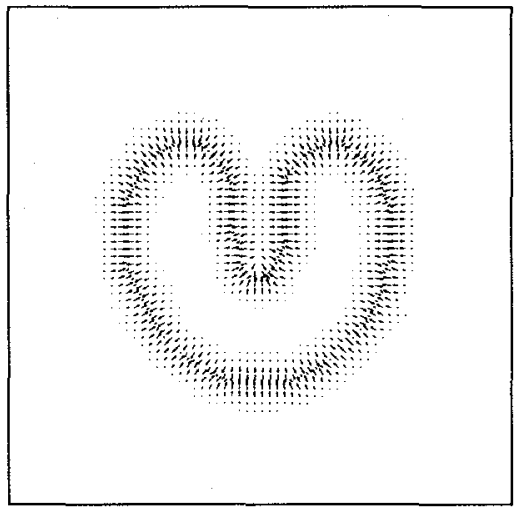

(b)
Figure 1. A snake with traditional potential forces cannot move into the concave boundary region.

the problem formulation, not its method of solution. In the following section, we introduce a new external force formulation which largely solves the problems discussed above.

\section{Gradient Vector Flow Field}

Our overall approach is to define a new non-irrotational external force field, which we call the gradient vector flow (GVF) field. Using a force balance condition as a starting point (rather than a variational formulation) we then let the GVF field replace the potential force field in (7), defining a new snake, which we call the GVF snake. The GVF field points toward the object boundary when very near to the boundary, but varies smoothly over homogeneous image regions, extending to the image border. The main advantages of the GVF field are that it can capture a snake from a long range - from either side of the object boundary — and can force it into concave regions.

\subsection{Edge Map}

We begin by defining an edge map $f(x, y)$ derived from the image $I(x, y)$ having the property that it is larger near the image edges ${ }^{2}$. Accordingly, we can use

$$
f(x, y)=-E_{\text {ext }}^{i}(x, y)
$$

where $i=1,2,3$, or 4 . The field $\nabla f$ has vectors pointing toward the edges, but it has a narrow capture range, in general. Furthermore, in homogeneous regions, $I(x, y)$ is constant, $\nabla f$ is zero, and therefore no information about nearby or distant edges is available.

\footnotetext{
${ }^{2}$ Other features besides edges can be sought by redefining $f(x, y)$ to be larger at desired features of interest, rather than edges.
}

\subsection{Gradient Vector Flow (GVF)}

We define the gradient vector flow (GVF) field to be the vector field $\mathbf{v}(x, y)=(u(x, y), v(x, y))$ that minimizes the energy functional

$\mathcal{E}=\iint \mu\left(u_{x}{ }^{2}+u_{y}{ }^{2}+v_{x}{ }^{2}+v_{y}{ }^{2}\right)+|\nabla f|^{2}|\mathbf{v}-\nabla f|^{2} d x d y$

This variational formulation follows a standard principle, that of making the result smooth when there is no data. In particular, we see that when $|\nabla f|$ is small, the energy is dominated by partial derivatives of the vector field, yielding a smooth field. On the other hand, when $|\nabla f|$ is large, the second term dominates the integrand, and is minimized by setting $\mathbf{v}=\nabla f$. The parameter $\mu$ is a regularization parameter governing the tradeoff between the first term and the second term. This parameter should be set according to the amount of noise present in the image (more noise, increase $\mu$ ).

We note that the smoothing term - the first term within the integrand of $(10)$ - is the same term used by Horn and Schunk in their classical formulation of optical flow [9]. On one hand, it is known that this term leads to the Laplacian operator in the corresponding Euler equations. On the other hand, it has recently been shown that this term corresponds to an equal penalty on the divergence and curl of the vector field [8]. Therefore, the external field resulting from this minimization can be expected to be neither entirely irrotational (as are the traditional snake potential fields) nor entirely solenoidal.

Using the calculus of variations [6], it can be shown that the GVF can be found by solving the following Euler equations

$$
\begin{aligned}
& \mu \nabla^{2} u-\left(u-f_{x}\right)\left(f_{x}{ }^{2}+f_{y}{ }^{2}\right)=0 \\
& \mu \nabla^{2} v-\left(v-f_{y}\right)\left(f_{x}{ }^{2}+f_{y}{ }^{2}\right)=0
\end{aligned}
$$

where $\nabla^{2}$ is the Laplacian operator. These equations give us another intuition behind the GVF formulation. We note that in homogeneous regions, the second term of both equations (11a) and (11b) is zero (because the gradient of $f(x, y)$ is zero). Therefore, within these regions, $u$ and $v$ are each determined by Laplace's equation. This results in a type of "filling-in" of information taken from the boundaries of the region.

Equations (11a) and (11b) can be solved by treating $u$ and $v$ as functions of time and solving

$$
\begin{aligned}
u_{t}(x, y, t)= & \mu \nabla^{2} u(x, y, t)-\left(u(x, y, t)-f_{x}(x, y)\right) \\
& \cdot\left(f_{x}(x, y)^{2}+f_{y}(x, y)^{2}\right) \\
v_{t}(x, y, t)= & \mu \nabla^{2} v(x, y, t)-\left(v(x, y, t)-f_{y}(x, y)\right) \\
& \cdot\left(f_{x}(x, y)^{2}+f_{y}(x, y)^{2}\right)
\end{aligned}
$$


The steady-state solution (as $t \rightarrow \infty$ ) of these linear parabolic equations is the desired solution of the Euler equations (11a) and (11b). Note that these equations are decoupled, and therefore can be solved as separate scalar partial differential equations in $u$ and $v$. The equations in (12) are known as generalized diffusion equations, and are known to arise in such diverse fields as heat conduction, reactor physics, and fluid flow [2]. For us, they have appeared from our description of desirable properties of external fields for active contours. Diffusion is a natural outcome given the desired "filling in" property. A stable explicit finite difference implementation for solving the steady-state solution of (12) was given in [19].

\subsection{GVF Snake}

After we compute $\mathbf{v}(x, y)$, we replace the potential force $-\nabla E_{\text {ext }}$ in the dynamic snake equation of (7) by $\mathbf{v}(x, y)$, yielding

$$
\mathbf{x}_{t}(s, t)=\alpha \mathbf{x}^{\prime \prime}(s, t)-\beta \mathbf{x}^{\prime \prime \prime \prime}(s, t)+\mathbf{v}
$$

We call the parametric curve solving the above dynamic equation as a GVF snake. This equation is solved in similar fashion to the traditional snake - i.e., by discretization and iterative solution.

We note that in [18], we generalized GVF to three dimensions and implemented a GVF deformable surface.

\section{GVF Fields and GVF Snakes}

This section shows several examples of GVF external field computations on simple objects as well as on one real medical image and demonstrates the performance of GVF snakes. The parameters $\mu$ (for GVF) and $\alpha$ and $\beta$ (for the snake) are specified in each case. All the edge map functions used for computing GVF are pre-normalized to the range $[0,1]$.

\subsection{Convergence to a Concave Region}

In our first experiment, we computed the GVF field for the line drawing of Fig. 2a using $\mu=0.2$. Comparing the resulting field, shown in Fig. $2 b$, to the potential force field of Fig. 1b, reveals several key differences. First, the GVF field has a much larger capture range. It is clear that in order to get this extent using traditional potential force fields, one would have to use a large $\sigma$ in the Gaussian filter. But this would have the effect of blurring (or perhaps even obliterating) the edges, which is not happening in the GVF field. A second observation is that the GVF vectors are pointing somewhat downward into the top of the U-shape, which should cause an active contour to move farther into this concave region. Finally, it is clear that the GVF field behaves

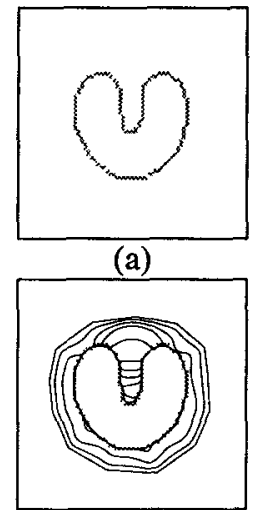

(c)

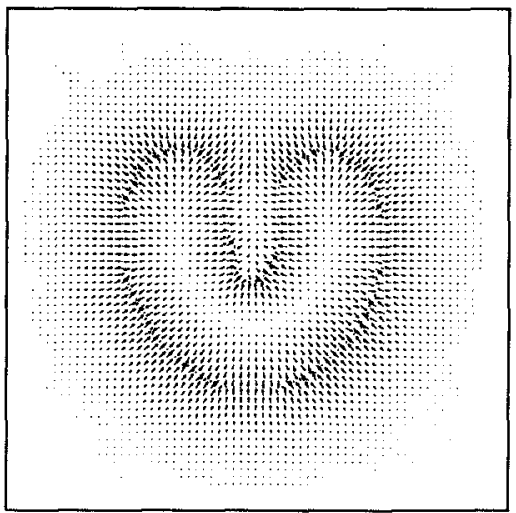

(b)
Figure 2. A snake with GVF external forces moves into the concave boundary region.

in an analogous fashion when viewed from the inside. That is, the vectors are pointing toward the boundary from as far away as possible and are pointing upward into the concave regions (the fingers of the U-shape) as viewed from the inside.

Fig. 2c shows the result of applying a GVF snake with parameters $\alpha=0.6$ and $\beta=0.0$ to the line drawing shown in Fig. $2 a$ (using the external GVF field of Fig. 2b). In this case, the snake was initialized farther away from the object than the initialization in Fig. 1c, and yet it converges very well to the boundary of the U-shape. It should be noted that the blocky appearance of the U-shape results from the fact that the image is only $64 \times 64$ pixels. The snake itself moves through the continuum (using bilinear interpolation to derive external field forces which are not at grid points) to arrive at a sub-pixel interpolation of the boundary.

\subsection{Streamlines of external force fields}

It is interesting to compare the capture range between the traditional potential force fields and GVF fields by looking at their streamlines. The streamlines are the paths over which a dense number of free particles move under the influence of external forces when placed in the external force field.

Fig. 3a gives the streamlines for the potential force field of Fig. 1b, and Fig. 3b gives the streamlines for the GVF field of Fig. 2b. Two effects are clear from this figure. First, the capture range of GVF is clearly much larger than that of the potential forces. Second, GVF provides downward forces within the concave region at the top of the U-shape, while potential forces only provide sideways forces. 


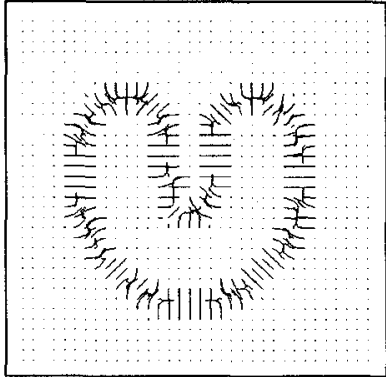

(a)

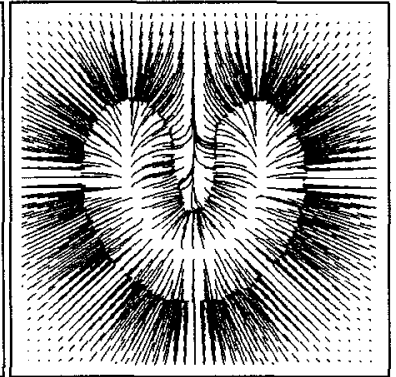

(b)
Figure 3. Stream lines of particles in (a) a potential force field and (b) a GVF field.

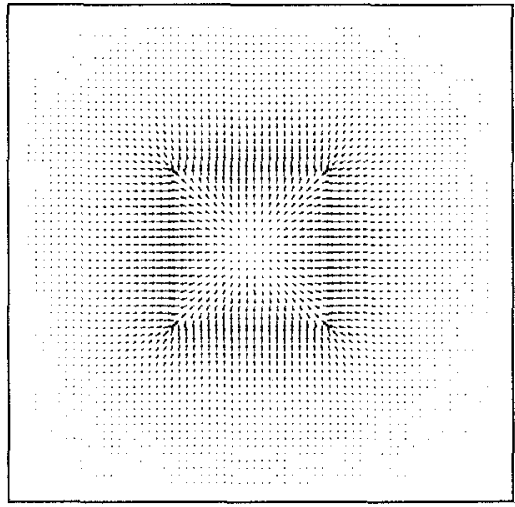

(a)

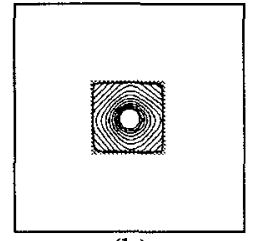

(b)

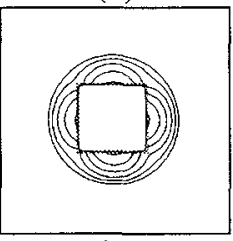

(c)
Figure 4. A GVF snake converges to the same result from either the inside or the outside.

\subsection{Snake Initialization}

Fig. 4a shows the computed GVF ( $\mu=0.1$ ) for the line drawing square shown using gray lines in Figs. $4 \mathrm{~b}$ and $4 \mathrm{c}$. Figs. $4 \mathrm{~b}$ and $4 \mathrm{c}$ show GVF snake results using initializations from the inside (Fig: 4b) and from the outside (Fig. 4c). The two final configurations are nearly indistinguishable from each other, indicating that the GVF snake can be initialized either inside or outside the desired boundary. It should be noted that, unlike pressure forces, the GVF snake does not require the a priori knowledge of whether to shrink or expand.

It can also be seen from Figs. $4 b$ and $4 c$ that the final configuration has slightly rounder corners than the square. This is one of the effects of $\mu$, the regularization parameter in the GVF formulation. Choosing $\mu$ smaller will tend to reduce this rounding, but will also reduce the strength of smoothing term. It should be noted, however, that this particular

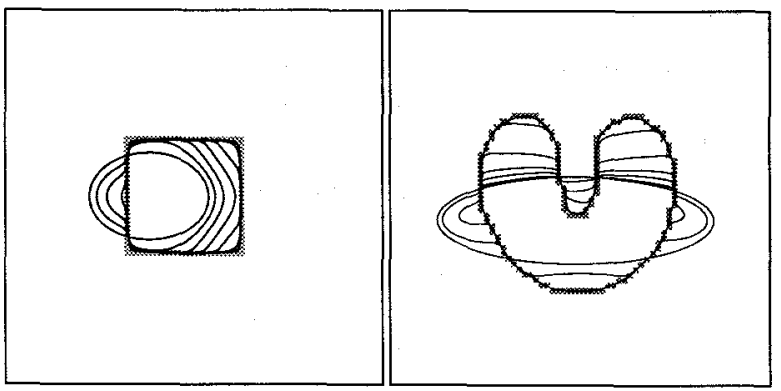

(a)

(b)

Figure 5. A GVF snake can also be initialized across the object boundary.

image has only $64 \times 64$ pixels, and the rounded corner of the snake is still within one pixel of the original corner.

Figs. $5 \mathrm{a}$ and $5 \mathrm{~b}$ demonstrate a further initialization insensitivity: the initial snake can cross the boundary. The result shown in Fig. 5a is nearly indistinguishable from that in Figs. $4 \mathrm{~b}$ and $4 \mathrm{c}$; and the result shown in Fig. $5 \mathrm{~b}$ is nearly indistinguishable from that shown in Fig. 2c. Of course, there must be limits on the full range of possible valid GVF snake initializations. A full theoretical and empirical study of these limits is a subject for future research.

\subsection{Gray-level Images}

The underlying formulation of GVF is valid for graylevel images as well as binary images. To compute GVF for gray-level images, the edge-map function $f(x, y)$ must first be calculated. Two possible choices for the edge-map are $f^{1}(x, y)=|\nabla I(x, y)|$ or $f^{2}(x, y)=\mid \nabla\left(G_{\sigma}(x, y) *\right.$ $I(x, y)) \mid$. A motivation for applying some Gaussian filtering to the underlying image is to reduce noise. Other more complicated noise-removal techniques such as median filtering, morphological filtering, and anisotropic diffusion could also be used to improve the underlying edge map. Given an edge-map function and an approximation to its gradient, GVF is computed in the usual way using Equation (12).

Fig. 6a shows a $(160 \times 160)$ magnetic resonance image (short-axis section) of the left ventrical of a human heart. Fig. $6 \mathrm{~b}$ shows an edge map computed using $f(x, y)=$ $f^{2}(x, y)$ with $\sigma=2.5$ (normalized to the range $[0,1]$ ). Fig. 6c shows the computed GVF, and Fig. 6d shows a sequence of GVF snakes (plotted in a shade of gray) and the GVF snake result (plotted in white), both plotted on the original image. Clearly, many details on the endocardial border are captured by the GVF snake result, including the papillary muscles (the bumps that protrude into the cavity). 


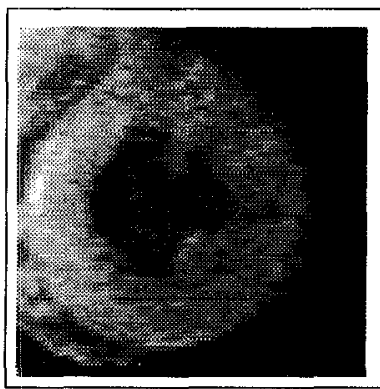

(a)

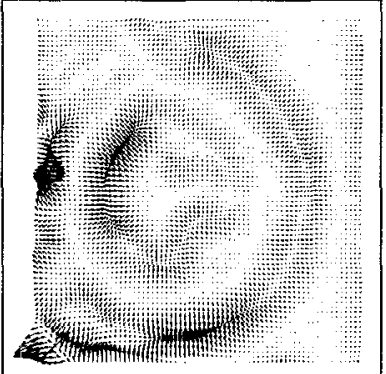

(c)

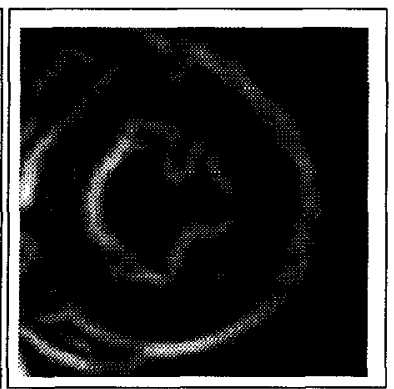

(b)

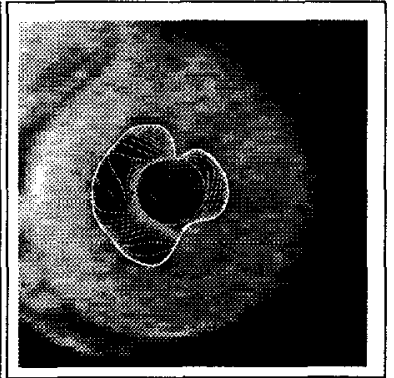

(d)
Figure 6. (a) A magnetic resonance image of the left ventrical of a human heart (short-axis section). (b) The edge map $\left|\nabla\left(G_{\sigma} * I\right)\right|^{2}$ with $\sigma=2.5$. (c) The computed GVF. (d) Initial and intermediate contours (gray curves) and the final contour (white curve) of the GVF snake.

\section{Conclusion}

We have introduced a new external force model for snakes called gradient vector flow (GVF). The field is calculated as a diffusion of the gradient vectors of a gray-level or binary edge map. We have shown that it allows for flexible initialization of the snake and encourages convergence to boundary concavities.

Further investigations into the nature and uses of GVF are warranted. A complete characterization of the capture range of the GVF field would help in snake initialization procedures. Investigations into the optimal selection of the GVF parameter $\mu$, and the interplay between $\mu$ and the snake parameters $\alpha$ and $\beta$ are desirable. Finally, the GVF framework might be useful in defining new connections between parametric and geometric snakes, and might form the basis for a new geometric snake.

\section{Acknowledgments}

The authors would like to thank Dzung Pham, Sandeep Gupta, and Prof. Joel Spruck for their discussions concerning this work.

\section{References}

[1] V. Caselles, F. Catte, T. Coll, and F. Dibos. A geometric model for active contours. Numerische Mathematik, 66:131, 1993.

[2] A. H. Charles and T. A. Porsching. Numerical Analysis of Partial Differential Equations. Prentice Hall, Engelwood Cliffs, NJ, 1990.

[3] I. Cohen, L. D. Cohen, and N. Ayache. Using deformable surfaces to segment 3-D images and infer differential structures. CVGIP: Image Understanding, 56(2):242-263, Sept. 1992.

[4] L. D. Cohen. On active contour models and balloons. CVGIP: Image Understanding, 53(2):211-218, Mar. 1991.

[5] L. D. Cohen and I. Cohen. Finite-element methods for active contour models and balloons for 2-D and 3-D images. IEEE Trans. on Pattern Anal. Machine Intell., 15(11):1131-1147, Nov. 1993.

[6] R. Courant and D. Hilbert. Methods of Mathematical Physics, volume 1. Interscience, New York, 1953.

[7] C. Davatzikos and J. L. Prince. An active contour model for mapping the cortex. IEEE Trans. on Medical Imaging, 14(1):65-80, Mar. 1995.

[8] S. N. Gupta and J. L. Prince. Stochastic models for DIVCURL optical flow methods. IEEE Signal Processing Letters, 3(2):32-35, 1996.

[9] B. K. P. Horn and B. G. Schunck. Determining optical flow. Artificial Intelligence, 17:185-203, 1981.

[10] M. Kass, A. Witkin, and D. Terzopoulos. Snakes: Active contour models. Int. J. Computer Vision, 1(4):321-331, 1987.

[11] B. Leroy, I. Herlin, and L. D. Cohen. Multi-resolution algorithms for active contour models. In 12th International Conference on Analysis and Optimization of Systems, pages $58-65,1996$.

[12] F. Leymarie and M. D. Levine. Tracking deformable objects in the plane using an active contour model. IEEE Trans. on Pattern Anal. Machine Intell., 15(6):617-634, 1993.

[13] R. Malladi, J. A. Sethian, and B. C. Vemuri. Shape modeling with front propagation: A level set approach. IEEE Trans. on Pattern Anal. Machine Intell., 17(2):158-175, 1995.

[14] J. L. Prince and C. Xu. A new external force model for snakes. In 1996 Image and Multidimensional Signal Processing Workshop, pages 30-31, 1996.

[15] H. Tek and B. B. Kimia. Image segmentation by reactiondiffusion bubbles. In Proc. Fifth Int. Conf. on Computer Vision, pages 156-162, 1995.

[16] D. Terzopoulos and K. Fleischer. Deformable models. The Visual Computer, 4:306-331, 1988.

[17] D. Terzopoulos and R. Szeliski. Tracking with Kalman snakes. In A. Blake and A. Yuille, editors, Active Vision, Artificial Intelligence, pages 3-20. The MIT Press, Cambridge, Massachusetts, 1992.

[18] C. Xu and J. L. Prince. Snakes, shapes, and gradient vector flow. IEEE Trans. on Image Processing. to appear.

[19] C. Xu and J. L. Prince. Snakes, shapes, and gradient vector flow. Technical Report JHU-ECE TR96-15, The Johns Hopkins University, Oct. 1996. 